\title{
InGaAs-QW VECSEL emitting >1300-nm via intracavity Raman conversion
}

\author{
Daniele C. Parrotta ${ }^{\mathrm{a}^{*}}$, Riccardo Casula ${ }^{\mathrm{a}}$, Jussi-Pekka Penttinen ${ }^{\mathrm{b}}$, Tomi Leinonen ${ }^{\mathrm{b}}$, Alan J. \\ Kemp ${ }^{\mathrm{a}}$, Mircea Guina ${ }^{\mathrm{b}}$, and Jennifer E. Hastie ${ }^{\mathrm{a}}$ \\ anstitute of Photonics, Department of Physics, University of Strathclyde, Technology and \\ Innovation Centre, 99 George Street, Glasgow G1 1RD, UK \\ ${ }^{\mathrm{b}}$ Optoelectronics Research Centre, Tampere University of Technology, Korkeakoulunkatu 3, \\ FIN-33101 Tampere, Finland
}

\begin{abstract}
We report intracavity Raman conversion of a long-wavelength InGaAs-QW VECSEL to $\sim 1320 \mathrm{~nm}$, the longest wavelength yet achieved by a VECSEL-pumped Raman laser. The setup consisted of a VECSEL capable of emitting $>17 \mathrm{~W}$ at $1180 \mathrm{~nm}$ and tunable from $1141-1203 \mathrm{~nm}$ and a 30-mm-long KGd( $\left(\mathrm{WO}_{4}\right)_{2}(\mathrm{KGW})$ Raman crystal in a coupled-cavity Raman resonator. The Raman cavity was separated from the VECSEL resonator by a tilted dichroic mirror, which steers the Raman beam to an output coupler external to the VECSEL. The spectral emission of the VECSEL, and consequently of the Raman laser, was set by a 4-mm-thick quartz birefringent filter in the VECSEL cavity. The KGW Raman laser was capable of emitting $2.5 \mathrm{~W}$ at $1315 \mathrm{~nm}$, with $\mathrm{M}^{2} \sim 2.7$ and $>4 \%$ diode-to-Stokes conversion efficiency. The Raman laser emission was tunable from 1295-1340 nm, limited by the free spectral range of the birefringent filter. Spectral broadening of the fundamental emission was observed during Raman conversion. At the maximum Raman laser output power, the total linewidth of the VECSEL spectrum was $\sim 0.7 \mathrm{~nm}$ FWHM. As a consequence, the Raman laser emission was also relatively broad $(\sim 0.9 \mathrm{~nm}$ FWHM). Narrow ( $<0.2 \mathrm{~nm}$ FWHM) Raman emission was obtained by inserting an additional $100 \mu \mathrm{m}$ etalon within the VECSEL cavity. With this configuration the fundamental intracavity power clamped at its value at the Raman threshold, suggesting an enhanced effective Raman gain, but the maximum output power of the Raman laser was $1.8 \mathrm{~W}$.
\end{abstract}

Keywords: Optically pumped semiconductor laser, Raman laser, tuning, VECSEL

\section{INTRODUCTION}

Vertical-external-cavity surface-emitting lasers (VECSELs) can emit several Watts in continuous-wave (CW) operation with good beam quality and low noise. With the insertion of an intracavity birefringent filter (BRF), VECSELs can also exhibit broad tunability $\left(>300 \mathrm{~cm}^{-1}[1]\right)$. VECSELs can emit over a broad range of wavelengths via bandgap engineering $[1,2]$; however there are certain spectral regions that cannot be easily accessed directly by VECSELs. The most mature VECSEL technology is based on InGaAs quantum wells (QWs), which are capable of efficient laser emission from $\sim 920-1180 \mathrm{~nm}[3-5]$. Due to their high intracavity fields, InGaAsbased structures are well-suited for intracavity nonlinear conversion, thus allowing laser operation in the visible via second harmonic generation [1,2] and the mid-infrared via optical parametric oscillation [6].

To date, VECSEL operation at $\sim 1300-1400 \mathrm{~nm}$ has been obtained either by adding nitrogen in the InGaAs lattice (e.g. GaInNAs QWs [7]) or using wafer-fused InAlGaAs/InP-based gain structures [8]. While dilute nitride structures are capable of emitting several Watts at $\sim 1200 \mathrm{~nm}$ [9], their performance drops significantly at longer wavelengths. On the other hand InP-based wafer-fused structures are capable of multi-Watt emission with high conversion efficiency ( $>50 \%$ in $[10]$ ), but their fabrication process is quite complex.

Here we propose Raman conversion of a long-wavelength $(\sim 1180 \mathrm{~nm})$ InGaAs VECSEL as an alternative way to achieve laser operation at $>1300 \mathrm{~nm}$. Intracavity-pumped Raman lasers are a convenient means to red-shift

* daniele.parrotta@strath.ac.uk 
the emission wavelength of several continuous wave lasers [11,12], including VECSELs [13-15]. The Stokes shift induced by stimulated Raman scattering is a fixed property of the Raman medium, and, unlike second-order nonlinearities, is an intrinsically phase-matched process. With the use of an intracavity wavelength filter (e.g. birefringent filter, etalon), the broad tunability of the VECSEL can be directly transferred to the Stokes shifted output.

\section{VECSEL GAIN STRUCTURE AND CHARACTERIZATION}

\subsection{Gain chip design}

The design of the $1180 \mathrm{~nm}$ VECSEL is similar to the one reported in $[5,16]$ and is illustrated in Figure 1. The gain chip semiconductor layer structure had 10 InGaAs/GaAs QWs with a nominal indium mole fraction of 37\%. The $7 \mathrm{~nm}$-thick quantum wells were located inside the active region at the anti-nodes of the optical standing wave. Relaxation of the highly-strained quantum wells was avoided by growing the QWs at a low growth temperature of $460^{\circ} \mathrm{C}$. Also, strain compensation layers, made of GaAsP a with phosphorus mole fraction of $11 \%$, were located between QWs and between the first QW and the DBR. The DBR had 25.5 pairs of alternating AlAs/GaAs layers. The gain chip was designed anti-resonant at the signal wavelength. On top of the active region, a $20 \mathrm{~nm}$ AlGaAs window layer and $10 \mathrm{~nm}$ GaAs capping layer were grown.

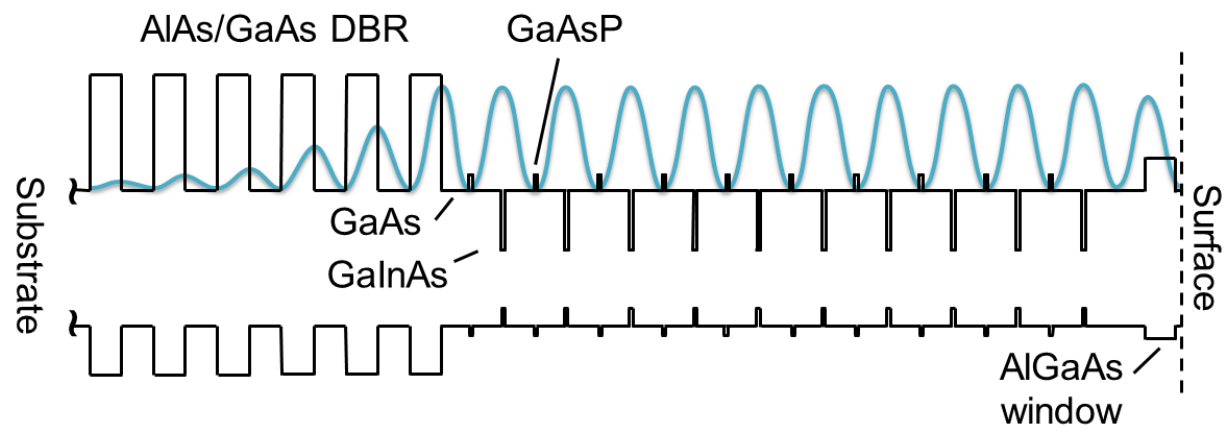

Figure 1. Schematic illustration of the VECSEL gain structure.

\subsection{Laser characterization}

The $1180 \mathrm{~nm}$ VECSEL chip was first tested in a 3-mirror cavity with a curved highly reflective (HR) folding mirror $(\mathrm{R}>99.98 \%$ at $1160-1200 \mathrm{~nm}, \mathrm{ROC}=200 \mathrm{~mm}$ ) and a flat output coupler (OC) as the end mirror (see Figure 2). The pump laser was a commercial $808 \mathrm{~nm}$ diode laser and its output beam was focused up to a $\sim 240 \mu \mathrm{m}$ radius spot on the gain chip with an angle of incidence of $\sim 40^{\circ}$. For thermal management, the gain chip was capillary-bonded to a wedged $\left(\sim 2^{\circ}\right) 300 \mu \mathrm{m}$-thick single-crystal diamond heatspreader. An antireflection coating designed for $1180 \mathrm{~nm}$ was deposited on the outer surface of the intracavity heatspreader. The distances between the elements were chosen to be: VECSEL-HR $=110 \mathrm{~mm}$ and HR-OC $=170 \mathrm{~mm}$. With $1.6 \%$ output

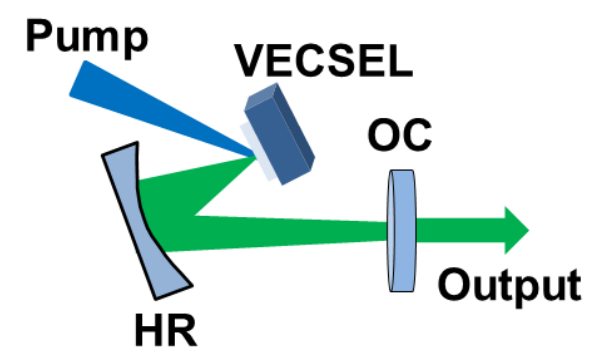

Figure 2. Schematic of the 3-mirror cavity VECSEL. HR: high reflectors. OC: output coupler. 
coupling and $10^{\circ} \mathrm{C}$ water coolant temperature, the VECSEL emitted up to $17.5 \mathrm{~W}$ for $88 \mathrm{~W}$ input power with $27 \%$ slope efficiency. With the insertion of a $2 \mathrm{~mm}$-thick quartz birefringent filter the laser emission was tunable from $1141.7-1202.7 \mathrm{~nm}$ (i.e. $61 \mathrm{~nm}$ tuning range) for an input pump power of $43 \mathrm{~W}$. The power transfer and the tuning curve are depicted in Figure 3. These results are comparable to the ones reported in [5].
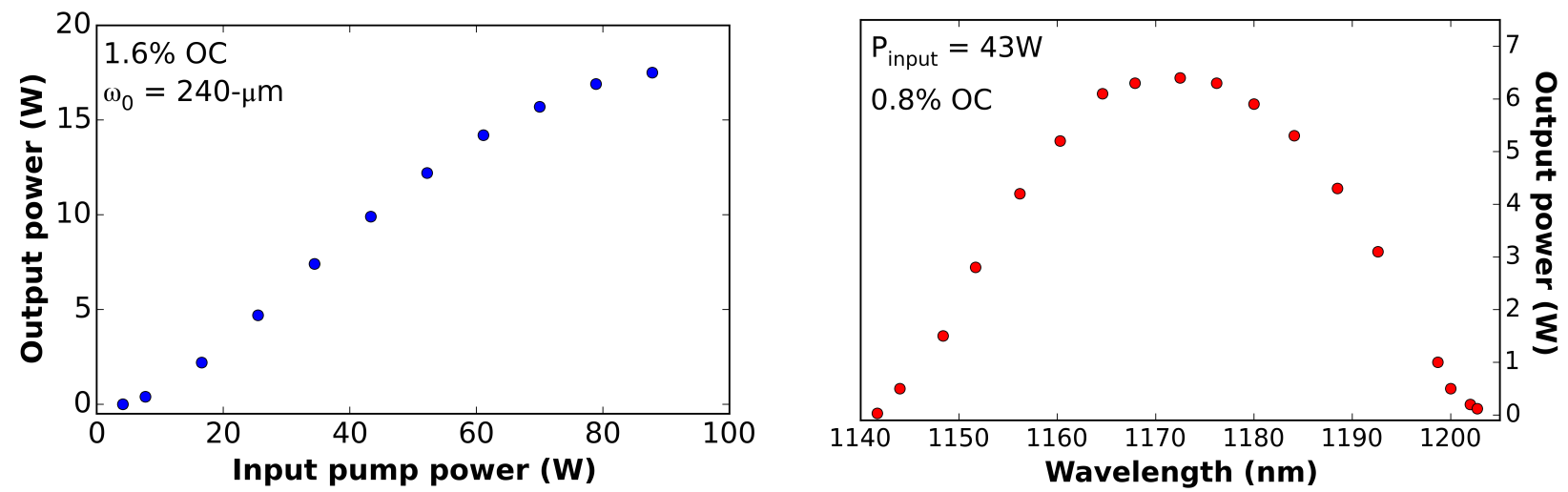

Figure 3. (Left) Power transfer characteristic of the 1180-nm VECSEL with $1.6 \%$ output coupling; (Right) Wavelength tuning at $43-\mathrm{W}$ input pump power using a $2-\mathrm{mm}$ quartz birefringent filter and a $0.8 \%$ output coupler.

\section{INTRACAVITY RAMAN CONVERSION}

\subsection{Experimental setup}

The experimental configuration of the VECSEL-pumped Raman laser is illustrated in Figure 4 . The $808 \mathrm{~nm}$ pump laser was focused up to $200 \mu \mathrm{m}$ radius onto the VECSEL gain chip. The Raman resonator was intracavitypumped within an all-high reflector $(\mathrm{R}>99.98 \%$ at $1150-1400 \mathrm{~nm})$ four-mirror VECSEL cavity. The Raman resonator was separated from the fundamental by a dichroic mirror with high reflection ( $>99.9 \%)$ at $>1290 \mathrm{~nm}$ and high transmission $(\mathrm{T}>99 \%)$ at $<1200 \mathrm{~nm}$, placed inside the cavity with a small tilt of $5^{\circ}$. The coupled cavity for the Raman field was completed by a plane output coupler (OC) mirror with transmission of $\sim 2.2 \%$ from 1300 to $1350 \mathrm{~nm}$. A $4 \mathrm{~mm}$-thick quartz birefringent filter was inserted at Brewster's angle within the VECSEL cavity, but outside the Raman resonator, to provide spectral narrowing and tuning of the VECSEL, and to pin the fundamental polarization. Both the resonators were aligned to produce a calculated $41 \mu \mathrm{m} \mathrm{TEM}_{00}$ waist radius at the centre of $30 \mathrm{~mm}$-long $\mathrm{KGd}\left(\mathrm{WO}_{4}\right)_{2}$ (KGW) Raman crystal. The KGW crystal was cut for beam propagation along the $\mathrm{N}_{p}$ axis and both end faces were antireflection coated for $950-1400 \mathrm{~nm}(\mathrm{R}<0.5 \%)$. The crystal was oriented for Raman excitation along the $\mathrm{N}_{m}$ axis to access the $901 \mathrm{~cm}^{-1}$ Stokes shift [17].

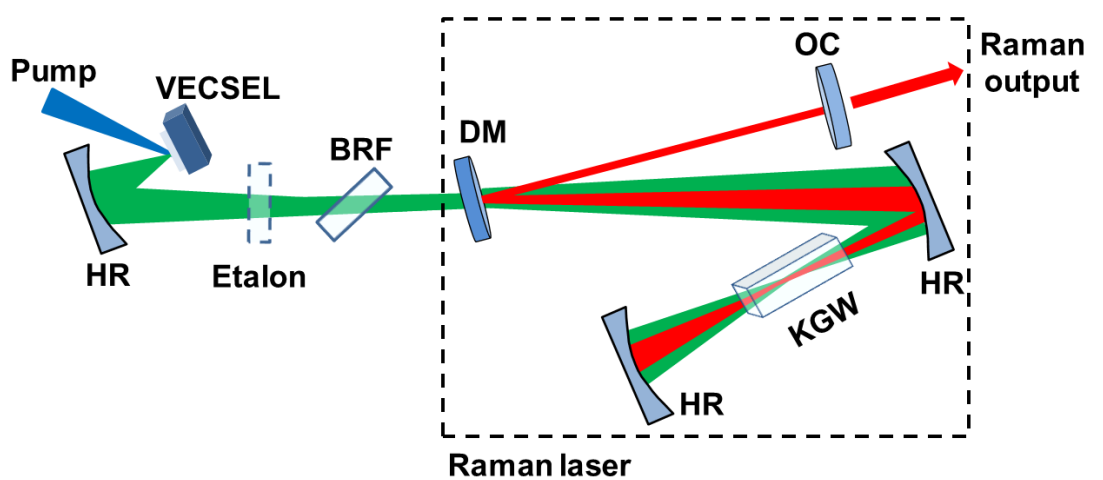

Figure 4. Schematic of the VECSEL-pumped Raman laser for $>1300 \mathrm{~nm}$. HR: high reflectors. BRF: birefringent filter. DM: dichroic mirror. OC: output coupler. The etalon plate was only utilized for single-frequency operation experiments. 


\subsubsection{Results}

Figure 5 (Left) shows the power transfers characteristics of the VECSEL-pumped KGW Raman laser with $2.2 \%$ output coupling. The Raman laser emitted up to $2.5 \mathrm{~W}$ at $1315 \mathrm{~nm}$ for an input pump power of $57 \mathrm{~W}$ ( $>4 \%$ diode-to-Stokes conversion efficiency). For higher pump powers, thermal rollover in the VECSEL led to lower output power of the Stokes-shifted field. The Raman laser threshold was achieved for an input pump power of $12.1 \mathrm{~W}$, when the VECSEL intracavity power was around $100 \mathrm{~W}$. Above the Raman threshold, the Stokes-shifted output power increased with pump power with a slope efficiency of 5.7\%. Raman conversion led to substantial spectral broadening of the VECSEL emission, and consequently of the Raman laser, as shown in Figure 5 (Right). This effect has been reported in several intracavity Raman laser systems $[12,14,18]$ where the fundamental gain bandwidth is larger than the Raman gain linewidth [18]. Due to their broad gain bandwidth, VECSELs are prone to spectral broadening during Raman conversion; however this can be prevented, or at least limited, by the presence of intracavity wavelength filters. It is important to notice that spectral broadening is actually detrimental for the performance of the Raman laser $[18,19]$. In an ideal intracavity Raman laser the fundamental intracavity power should clamp at its value at the Raman threshold [20], but here the VECSEL intracavity power keeps increasing above Raman threshold. The fundamental intracavity power nearly clamps only for input pump powers greater than $30 \mathrm{~W}$, when the VECSEL and the Raman spectra have similar emission linewidths. At the maximum output power the emission linewidths of the VECSEL and the Raman were measured to be $\sim 0.7 \mathrm{~nm}$ $\left(\sim 5 \mathrm{~cm}^{-1}\right)$ and $\sim 0.9 \mathrm{~nm}\left(\sim 5 \mathrm{~cm}^{-1}\right) \mathrm{FWHM}$, respectively. It is interesting to notice that both spectra show several peaks, but apparently they are not related to an etalon effect as in [14]. As a matter of fact, the observed peak separation of $\sim 0.55 \mathrm{~nm}$ does not correspond to an etalon effect due to the heatspreader or the dichroic mirror, although we do not rule out they may contribute somehow. We note, however, that spectral broadening with multi-peak emission has also been reported in [18]. The beam quality factors of the Raman laser and the VECSEL at maximum output power were measured to be $\sim 2.7$ and $>3$, respectively. Beam quality degradation is commonly observed in intracavity Raman lasers and contributes to the suppression of the expected clamping of the fundamental intracavity power $[14,19]$.

Wavelength tuning of the VECSEL, and consequently of the Raman laser, was achieved by rotating the birefringent filter. The VECSEL emission was tuned from 1160 to $1196 \mathrm{~nm}$, limited by the free spectral range of the $4 \mathrm{~mm}$-thick birefringent filter. Figure 6 shows that the Raman laser was tunable from 1295 to $1340 \mathrm{~nm}$ with $\sim 2.2 \%$ output coupling. The whole tuning range of the VECSEL was Stokes shifted. During Raman conversion, the VECSEL and the Raman spectra were both broadened $\left(\sim 5 \mathrm{~cm}^{-1} \mathrm{FWHM}\right)$ over the full tuning range.

\subsubsection{Single-frequency operation}

To reduce the spectral broadening of the fundamental emission during Raman conversion, we inserted an uncoated $100 \mathrm{~mm}$-thick Suprasil etalon plate in the VECSEL cavity, outside the Raman resonator. The free spectral range of
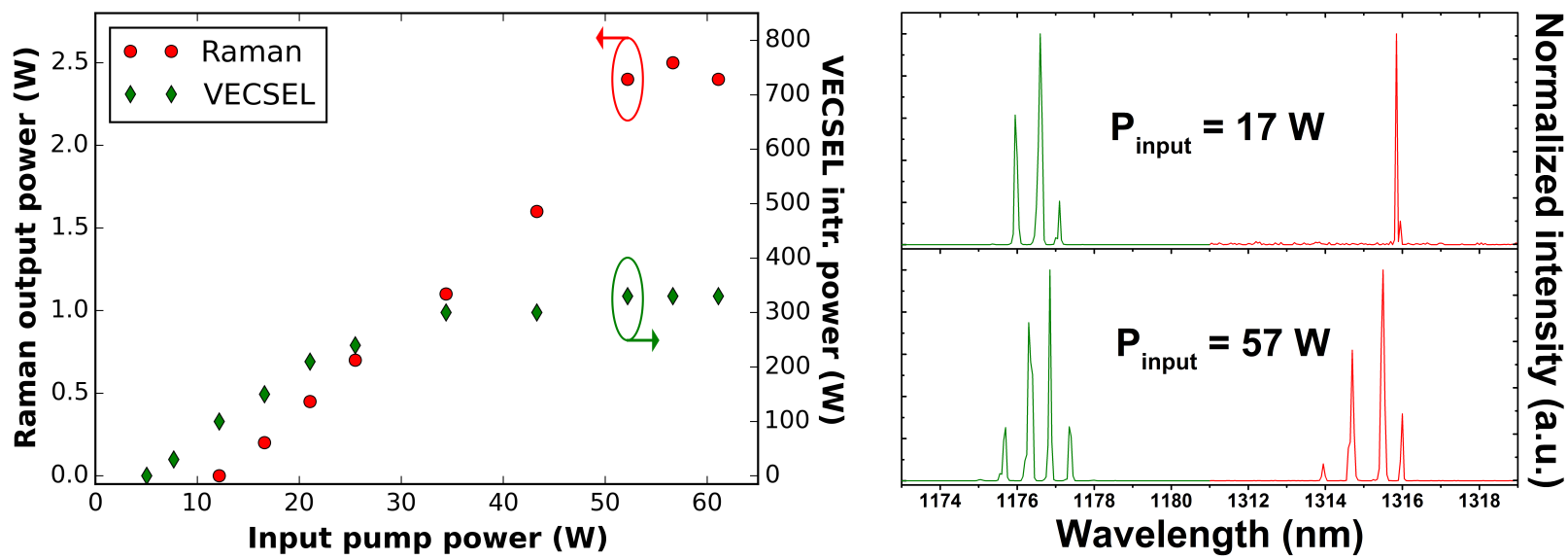

Figure 5. (Left) Power transfer characteristics of the Raman laser (red circles) and the VECSEL (green diamonds) for $2.2 \%$ output coupling at $1315 \mathrm{~nm}$; (Right) VECSEL (green line) and Raman laser (red line) spectral emissions near Raman threshold and at the maximum output power. 


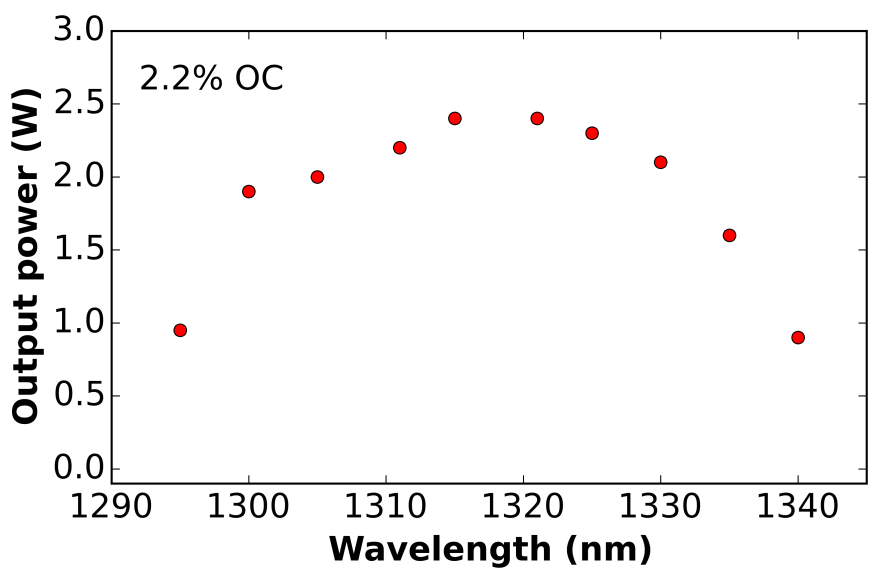

Figure 6. Raman laser tuning curve for an input pump power of $57 \mathrm{~W}$ with $2.2 \%$ output coupling.

this etalon is calculated to be $\sim 4.8 \mathrm{~nm}$, which proved to be enough to suppress fundamental spectral broadening. Figure 7 shows the power transfers and the spectral characteristics of the VECSEL and the Raman laser using this configuration. Both lasers emitted a single narrow peak, with a measured linewidth of $<0.2 \mathrm{~nm}\left(<0.15 \mathrm{~cm}^{-1}\right)$ FWHM. The slope efficiency of the Raman emission was calculated to be $6.7 \%$. Raman threshold was achieved for an intracavity fundamental power of $\sim 100 \mathrm{~W}$ (as in the other system), but above Raman threshold it only increased slightly, up to $\sim 115 \mathrm{~W}$. This suggests that with the insertion of the etalon plate, the effective Raman gain $\left(g_{e} \sim A_{e} / P_{f}\right.$, where $A_{e}$ and $P_{f}$ are the effective area for Raman conversion and the fundamental intracavity power, respectively - see Refs. $[15,19])$ above the Raman threshold is higher than in the previous configuration (up to 3 times if $A_{e}$ is unchanged). This is consistent with the results reported in [18]. However, despite the larger effective Raman gain, the maximum output power of the Raman laser was limited to $1.8 \mathrm{~W}$. We note that with the insertion of the etalon plate thermal rollover occurs at lower pump power $(\sim 45-50 \mathrm{~W})$ than without it $(>57 \mathrm{~W})$, hinting that the VECSEL losses have increased. Besides, despite the fact that the fundamental intracavity power clamped, here the slope efficiency of the Raman laser is nearly unchanged $(\sim 6.7 \%$, while in the other setup is 5.7\%); a possible explanation is that the increased losses in the VECSEL led to a reduction in its internal efficiency [21], thus affecting the slope efficiency of the Raman laser [14,19].
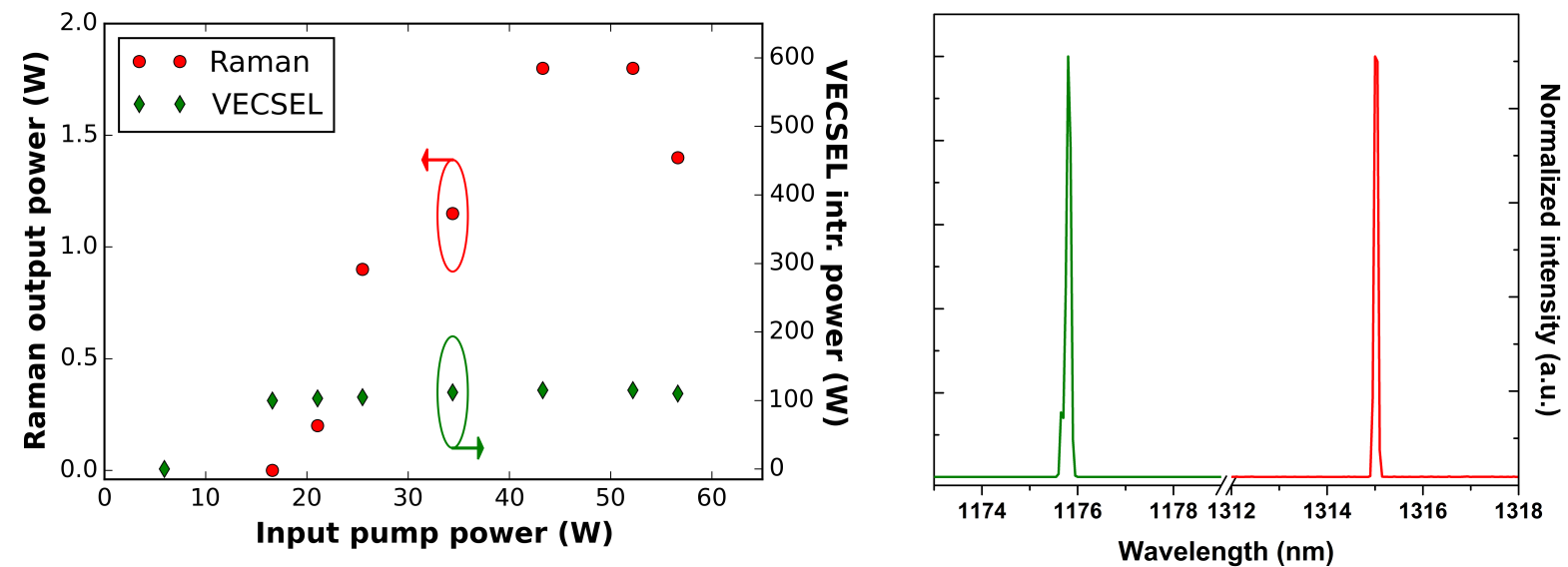

Figure 7. (Left) Power transfer characteristics of the Raman laser (red circles) and the VECSEL (green diamonds) for $2.2 \%$ output coupling at $1315 \mathrm{~nm}$; (Right) VECSEL (green line) and Raman laser (red line) spectral emissions at the maximum output power. 


\section{CONCLUSION}

We report intracavity Raman conversion of an $1180 \mathrm{~nm}$ InGaAs VECSEL. Output power up to $2.5 \mathrm{~W}$ at $1315 \mathrm{~nm}$, with diode-to-Stokes conversion efficiency $>4 \%, \mathrm{M}^{2} \sim 2.7$, and relatively broad emission $\left(\sim 5 \mathrm{~cm}^{-1} \mathrm{FWHM}\right)$ was achieved. The Raman laser was tunable from 1295 to $1340 \mathrm{~nm}$, limited by the free spectral range of the birefringent filter. With the insertion of an additional etalon plate, the Raman laser emitted up to $1.8 \mathrm{~W}$, with narrow emission linewidth $\left(<1.5 \mathrm{~cm}^{-1} \mathrm{FWHM}\right)$. Intracavity Raman conversion extends the spectral coverage of InGaAs VECSELs beyond $1300 \mathrm{~nm}$. Work is ongoing to improve the performance of the reported system.

\section{ACKNOWLEDGMENTS}

This work was supported by the Engineering and Physical Sciences Research Council (EPSRC), UK, under grant EP/I022791/1, the Academy of Finland project Qubit (decision \#278388) and TEKES project ReLase (40016/14). Riccardo Casula acknowledges a studentship part funded by the EPSRC and the European Research Council (ERC, grant number 278389). Alan J. Kemp acknowledges support from the ERC, the Royal Academy of Engineering and Fraunhofer UK Research Ltd.

\section{REFERENCES}

[1] Okhotnikov (editor), O., [Semiconductor Disk Lasers: Physics and Technology], Wiley-VCH (2010).

[2] Calvez, S., Hastie, J. E., Guina, M., Okhotnikov, O. G., and Dawson, M. D., "Semiconductor disk lasers for the generation of visible and ultraviolet radiation," Laser 83 Photonics Reviews 3, 407-434 (Sept. 2009).

[3] Hein, A., Demaria, F., Kern, A., Menzel, S., Rinaldi, F., Rosch, R., and Unger, P., "Efficient 460-nm second-harmonic generation with optically pumped semiconductor disk lasers," IEEE Photonics Technology Letters 23, 179-181 (Feb. 2011).

[4] Heinen, B., Wang, T.-L., Sparenberg, M., Weber, A., Kunert, B., Hader, J., Koch, S., Moloney, J., Koch, M., and Stolz, W., "106 W continuous-wave output power from vertical-external-cavity surface-emitting laser," Electronics Letters 48, 516-517 (Apr. 2012).

[5] Ranta, S., Tavast, M., Leinonen, T., Van Lieu, N., Fetzer, G., and Guina, M., "1180 nm VECSEL with output power beyond 20 W," Electronics Letters 49, 59-60 (Jan. 2013).

[6] Stothard, D. J. M., Hopkins, J.-M., Burns, D., and Dunn, M. H., "Stable, continuous-wave, intracavity, optical parametric oscillator pumped by a semiconductor disk laser (VECSEL)," Optics Express 17, 1064810658 (June 2009).

[7] Hopkins, J.-M., Smith, S., Jeon, C., Sun, H., Burns, D., Calvez, S., Dawson, M., Jouhti, T., and Pessa, M., "0.6 W CW GaInNAs vertical external-cavity surface emitting laser operating at $1.32 \mu \mathrm{m}$," Electronics Letters 40, 30-31 (Jan. 2004).

[8] Lyytikäinen, J., Rautiainen, J., Toikkanen, L., Sirbu, A., Mereuta, A., Caliman, A., Kapon, E., and Okhotnikov, O. G., "1.3- $\mu \mathrm{m}$ optically-pumped semiconductor disk laser by wafer fusion," Optics Express 17, 9047-9052 (May 2009).

[9] Korpijärvi, V.-M., Leinonen, T., Puustinen, J., Härkönen, A., and Guina, M. D., "11 W single gain-chip dilute nitride disk laser emitting around 1180 nm," Optics Express 18, 25633-25641 (Dec. 2010).

[10] Keller, S., Sirbu, A., Iakovlev, V., Caliman, A., Mereuta, A., and Kapon, E., "8.5 W VECSEL output at $1270 \mathrm{~nm}$ with conversion efficiency of $59 \%$," Optics Express 23, 17437-17442 (June 2015).

[11] Pask, H. M., "Continuous-wave, all-solid-state, intracavity Raman laser," Optics Letters 30(18), 2454-2456 (2005).

[12] Fan, L., Fan, Y., Li, Y., Zhang, H., Wang, Q., Wang, J., and Wang, H., "High-efficiency continuous-wave Raman conversion with a BaWO4 Raman crystal," Optics Letters 34, 1687-1689 (June 2009).

[13] Parrotta, D. C., Lubeigt, W., Kemp, A. J., Burns, D., Dawson, M. D., and Hastie, J. E., "Continuous-wave Raman laser pumped within a semiconductor disk laser cavity," Optics Letters 36, 1083-1085 (Apr. 2011).

[14] Parrotta, D. C., Kemp, A. J., Dawson, M. D., and Hastie, J. E., "Multiwatt, continuous-wave, tunable diamond Raman laser with intracavity frequency-doubling to the visible region," IEEE Journal of Selected Topics in Quantum Electronics 19, 1400108-1400108 (July 2013). 
[15] Schlosser, P. J., Parrotta, D. C., Savitski, V. G., Kemp, A. J., and Hastie, J. E., "Intracavity Raman conversion of a red semiconductor disk laser using diamond," Optics Express 23, 8454-8461 (Apr. 2015).

[16] Kantola, E., Leinonen, T., Ranta, S., Tavast, M., and Guina, M., "High-efficiency 20 W yellow VECSEL," Optics Express 22, 6372-6380 (March 2014).

[17] Mochalov, I. V., "Laser and nonlinear properties of the potassium gadolinium tungstate laser crystal $\mathrm{KGd}\left(\mathrm{WO}_{4}\right)_{2}: \mathrm{Nd}^{3+}{ }_{-}(\mathrm{KGW}: \mathrm{Nd}), "$ Optical Engineering 36, 1660-1669 (June 1997).

[18] Bonner, G. M., Lin, J., Kemp, A. J., Wang, J., Zhang, H., Spence, D. J., and Pask, H. M., "Spectral broadening in continuous-wave intracavity Raman lasers," Optics Express 22, 7492-7502 (Apr. 2014).

[19] Spence, D. J., "Spatial and spectral effects in continuous-wave intracavity Raman lasers," IEEE Journal of Selected Topics in Quantum Electronics 21, 134-141 (Jan. 2015).

[20] Spence, D. J., Dekker, P., and Pask, H. M., "Modeling of continuous wave intracavity Raman lasers," IEEE Journal of Selected Topics in Quantum Electronics 13, 756 -763 (May-June 2007).

[21] Maclean, A. J., Birch, R. B., Roth, P. W., Kemp, A. J., and Burns, D., "Limits on efficiency and power scaling in semiconductor disk lasers with diamond heatspreaders," J. Opt. Soc. Am. B 26, 2228-2236 (Dec. 2009). 